REGARDS

SUR L'ECONOMIE ALLEMAND

BULLETIN ECONOMIQUE DU CIRAC
Regards sur l'économie allemande

Bulletin économique du CIRAC

$88 \mid 2008$

Varia

\title{
SMIC légal : une loi toujours en projet
}

Isabelle Bourgeois

\section{OpenEdition}

\section{Journals}

Édition électronique

URL : http://journals.openedition.org/rea/1573

DOI : 10.4000/rea.1573

ISBN : 978-2-8218-0872-0

ISSN : 1965-0787

Éditeur

CIRAC

Édition imprimée

Date de publication : 1 octobre 2008

Pagination : 35-37

ISSN : 1156-8992

Référence électronique

Isabelle Bourgeois, «SMIC légal : une loi toujours en projet », Regards sur l'économie allemande [En ligne], 88 | octobre 2008, mis en ligne le 01 octobre 2010, consulté le 15 septembre 2020. URL : http:// journals.openedition.org/rea/1573

Ce document a été généré automatiquement le 15 septembre 2020.

(C) CIRAC 


\title{
SMIC légal : une loi toujours en projet
}

\author{
Isabelle Bourgeois
}

\section{Deux projets de loi bientôt en débat parlementaire}

1 En plein mois de juillet, le conseil des ministres fédéral a adopté, en application d'un accord conclu le 18 juin par les partenaires de la coalition (SPD et CDU/CSU), deux projets de loi qui, dans une articulation complémentaire, visent à instituer un salaire minimum légal dans certaines branches et secteurs d'activité. Les deux textes, en date du 16-07-2008, seront soumis à première lecture au Bundestag le 16 octobre et au Bundesrat pour une dernière lecture et en principe adoption définitive le 19 décembre.

2 La précipitation des événements dans le secteur de la finance en ce début d'octobre ayant relégué à l'arrière-plan des préoccupations les débats autour du SMIC légal où s'opposent depuis de longs mois les formations du gouvernement de coalition (le ministre fédéral du Travail est SPD, son collègue de l'Economie, CSU), mais aussi les acteurs économiques et le monde syndical ou encore certains Länder. Les positions antagonistes ne s'en affrontent pas moins vivement. Au point que rien ne permet d'affirmer, en ce début d'octobre, que les projets de loi seront effectivement adoptés en l'état.

\section{Deux textes amendant...}

Dans les deux cas, comme les possibilités de déroger au principe constitutionnel de l'autonomie tarifaire des partenaires sociaux sont très limitées en Allemagne, il s'agit d'amendements apportés à deux des trois seuls textes existants qui permettent à l'Etat de se substituer aux partenaires sociaux en réglementant par la loi ce qui est normalement régulé par la voie du contrat. 


\section{... la Loi sur les travailleurs détachés (AEntG)...}

4 Le premier projet d'amendement est celui de la Loi sur les travailleurs détachés (Arbeitnehmer-Entsendegesetz, AEntG) qui avait été votée en 1996 en prévision de l'adoption de la Directive 71/96/CE sur les travailleurs détachés. Son objectif étant à l'époque de protéger les salariés du BTP est-allemand contre la concurrence de la maind'œuvre est-européenne, cette loi avait introduit un salaire minimum horaire obligatoire dans plusieurs métiers du BTP (au moins $7 €$ ). Son domaine d'application avait ensuite été étendu en 2006 à pratiquement l'ensemble des activités du BTP puis, à

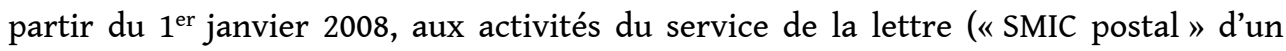
montant allant jusqu'à 9,80 €/heure ; voir REA 85/08).

$5 \quad$ Après une procédure de candidature des branches intéressées par un SMIC, close le 31 mars dernier, le périmètre des secteurs concernés par la Loi AEntG avait été étendu. La candidature était ouverte à toute branche dont plus de $50 \%$ des effectifs sont couverts par une convention salariale, ceci afin de respecter l'autonomie tarifaire des partenaires sociaux et d'établir le futur SMIC sur la base des salaires planchers en vigueur.

6 Le projet de loi de réforme de la Loi $A E n t G$ en discussion couvre donc, outre les métiers du BTP et de la lettre, les 7 domaines d'activité suivants: intérim, prestations dépendance, sécurité, teinturerie, formation continue, services des forêts et certaines activités minières. Si en l'état actuel, la Loi $A E n t G$ s'applique à 1,8 millions de salariés, s'y ajouteraient après adoption du nouveau texte 1,43 millions de personnes, estime le ministère fédéral du Travail (communiqué, 16-07-08).

7 Outre l'extension du périmètre des activités concernées, le texte de loi en débat prévoit que les SMIC légaux fixés dans les divers secteurs concernés s'appliqueront sans distinction de nationalité ou de provenance à tous les salariés occupés sur le sol allemand, ce qui généralise le principe européen du pays d'accueil aux secteurs auxquels ne s'applique pas la Directive services régie, elle au contraire, par le principe du pays d'origine.

\section{... et la Loi sur les conditions de travail minimales (Mia)}

8 Le second projet est une nouvelle version de la Loi sur les conditions de travail minimales (Mindestarbeitsbedingungengesetz, Mia) de 1952 qui permet au législateur de se substituer aux partenaires sociaux en cas de défaillance de ceux-ci, c'est-à-dire en ultime recours seulement. Elle n'a jamais eu à être appliquée en plus d'un demi siècle.

9 Le projet d'amendement prévoit que l'Etat légiférera sur les salaires dès lors que, dans un secteur donné, aucune convention n'a été conclue ou si une telle convention ne s'applique qu'à moins de $50 \%$ des salariés d'un secteur régulé par voie de convention. La fixation d'un SMIC légal est donc ici fonction du degré de couverture conventionnelle constaté et vise à réglementer les secteurs d'activité échappant à la logique traditionnelle de branche (ils résident principalement dans le tertiaire).

10 Le domaine d'application du projet de loi intègre sans le dire le principal objectif de la procédure d'extension (Allgemeinverbindlichkeitserklärung) prévue par la Loi sur les conventions tarifaires (Tarifvertragsgesetz) de 1949, modifiée pour la dernière fois en 2006 : à savoir intervenir, dans l'intérêt supérieur de la cohésion sociale, notamment en 
cas de risque de fracture sociale découlant d'un trop important différentiel salarial dans un secteur donné. Or le recours à cette disposition est une pratique très marginale dans le système conventionnel allemand. Dès lors, dans le projet de réforme de la Loi Mia, la situation "d'urgence sociale» se trouve étendue à l'ensemble des activités puisque, du fait de la structure comme du poids même des activités concernées dans l'économie (la plupart des services), la référence en matière de salaires n'est plus un seule branche donnée avec ses normes mais, à l'inverse, l'ensemble des branches régies par convention - donc en réalité la quasi-totalité de l'industrie.

11 Concrètement, à l'image de la procédure d'extension, les décisions en matière de SMIC sont prises par une commission, mais avec une différence : cette nouvelle commission sera instituée à demeure et non pas rassemblée ad hoc. Comme dans le cadre de la procédure d'extension, les salaires minima seront promulgués par décret du gouvernement fédéral. Le montant ainsi fixé du SMIC sera contraignant et s'appliquera à tous les salariés concernés sur le sol allemand, qu'ils soient Allemands ou étrangers.

\section{Objectif des deux textes : hausser les minima salariaux}

12 Ces deux projets de loi, présentés par le ministère fédéral du Travail (SPD) comme un élément central "pour un travail meilleur et plus juste» et "pour des salaires plus équitables ", vise expressément à créer des seuils minima dans le segment à bas salaires, "caractérisé par une forte proportion de femmes et un degré d'organisation inférieur à la moyenne ", ainsi que le formule l'exposé des motifs. Ils s'appliqueront de facto, s'ils sont votés, à la majorité des salariés du tertiaire, un secteur que le syndicat des services Ver.di ne parvient pas à organiser. Si aucun seuil n'est bien sûr encore fixé, les syndicats du DGB plaident pour 7,50€ de l'heure.

\section{De très vives critiques}

13 Ces projets de loi s'attirent de vives critiques (voir REA 85/08 et 86/08), qui portent sur deux points principalement: la conformité avec le principe constitutionnel de l'autonomie tarifaire, et les impacts sur l'économie du seuil salarial en débat.

\section{Des textes conformes ou non à la Constitution?}

Sous l'angle du droit constitutionnel, la large extension des prérogatives que la Loi fondamentale n'accorde à l'Etat (législateur) qu'en ultime recours s'assimile à une violation du principe de la liberté de coalition garanti par l'art.9 de la Loi fondamentale. C'est ce droit fondamental qui fonde l'autonomie conventionnelle des partenaires sociaux au niveau de leur branche (voir REA 52-53) ; une autonomie fondée sur le principe de subsidiarité. Les questions juridiques soulevées par le projet d'instauration d'un SMIC légal sont importantes, amplement débattues par les constitutionnalistes, et elles occupent d'ores et déjà la juridiction. Le 7 mars dernier, le tribunal administratif de Berlin, saisi divers concurrents de Deutsche Post AG, avait invalidé le SMIC postal. En réaction, le ministre fédéral du Travail avait interjeté appel 
auprès de l'instance supérieure. L'affaire est toujours en instance, et il n'est pas exclu que le Tribunal constitutionnel soit saisi.

15 Parmi ceux qui plaident pour un retrait des textes en projet figurent aussi bien des représentants des milieux économiques que politiques, mais également certains Länder comme le Bade-Wurtemberg et, au sein du Bundesrat, la Commission Travail et politique sociale. A l'opposé, le SPD, Ver.di et plus généralement le milieu syndical estiment que le «rétablissement de la justice sociale » légitime, en tant qu'intérêt supérieur, l'intervention de l'Etat dans un domaine réservé à la société civile organisée.

\section{Quel impact sur l'économie?}

Sous l'angle de l'impact d'un SMIC légal sur l'économie, les arguments sont plus diversifiés, mais se laissent classer en trois principales catégories, relatives d'une part à la finalité ou à l'opportunité de la mesure, d'autre part, au seuil envisagé et, enfin, aux effets sur la situation globale de l'emploi. Dans la première catégorie, marquée par la dichotomie entre "salaire juste, équitable pour tous" et "nécessaire flexibilité salariale » ou "efficacité économique », et nourrie par les problématiques du pouvoir d'achat ou de la précarité, s'affrontent bien évidemment SPD et syndicats (Ver.di en tête) d'un côté, milieux économiques (BDA, BDI) et CDU/CSU de l'autre. Les arguments des uns et des autres sont les mêmes de part et d'autre du Rhin.

\section{Quel seuil choisir, le cas échéant?}

17 Les arguments avancés en ce qui concerne le seuil des $7,50 € / \mathrm{h}$ plaidés par le DGB sont nettement plus complexes. Les adversaires les plus farouches, c'est-à-dire la quasitotalité des fédérations de l'économie, ainsi que la majorité des économistes, opposent un franc refus de toute fixation d'un seuil plancher par la loi. Ils estiment en effet que non seulement, une telle mesure, surtout si elle est généralisée, détruirait l'emploi, mais que, de surcroît, elle est inutile. Car de tels seuils existent déjà (ce sont les minima sociaux constitués par l'aide sociale et le forfait de base de l'allocation Hartz IV) et ceux-ci créent de facto un salaire minimum implicite de référence. Or les réformes prises dans le cadre des lois Hartz avaient justement abaissé ces seuils afin de mettre fin aux trappes à inactivité qui étaient et sont encore une des causes du haut niveau de chômage structurel en Allemagne (voir REA 68/04) et avaient permis d'ouvrir la fourchette salariale vers le bas - ce que les syndicats, pourtant co-signataires des accords préalables aux réformes Hartz, considèrent aujourd'hui comme une "précarisation ». Moins opposés sur le principe, certains économistes comme Bernd Rürup, membre du Conseil des Sages, pensent que rien ne s'oppose à un seuil plancher légal explicite, à condition que celui-ci soit suffisamment bas (4,50€ par exemple) pour ne pas interférer dans la fixation des salaires par le marché. Mais, lui rétorquent d'autres experts, rien ne garantit que, un tel seuil une fois fixé, il ne donne pas matière à surenchère en période électorale.

La question du montant à retenir divise même le camp syndical : le puissant district de Rhénanie du Nord-Westphalie du syndicat IG Metall considère depuis toujours que $7,50 € / h$ présentent un risque de dévaluation pour les salaires planchers en vigueur dans la métallurgie, et qui sont nettement plus élevés. La métallurgie perdrait donc un 
avantage compétitif notable en comparaison des services. Plus récemment (début septembre), le bureau du DGB a fait part à la presse de sa préoccupation : les projets de loi ne permettraient pas l'institution de salaires garantissant un « minimum vital». Et la confédération réclame une modification des textes afin que rien ne permette de prendre pour référence les seuils de «dumping" pratiqués par la fédération (concurrente) des syndicats chrétiens ou toutes les nouvelles organisations professionnelles et catégorielles qui se multiplient outre-Rhin, bouleversant le champ syndical (voir REA 83/07).

\section{Les enjeux : lutte d'influence entre représentations syndicales...}

On voit bien là quels sont, indépendamment du contexte (pré)électoral, les enjeux réels des débats toujours en cours sur l'adoption d'un SMIC légal en Allemagne. Il s'agit d'une lutte d'influence entre des syndicats en perte de représentativité (IG Metall, par exemple), d'autres qui ne sont jamais parvenus à organiser en branche les nouvelles activités issues de la tertiarisation (Ver.di), et d'autres encore, qui montent en puissance, et qui ne visent que la défense d'intérêts catégoriels ou professionnels (conducteurs de trains, pilotes, etc.). Le pivot de cette guerre est la "justice salariale » qu'incarnerait un minimum élevé garanti à tous. Et comme dans toute guerre, les moyens employés ne sont pas toujours les plus purs.

\section{... qui 'surfent' sur la question du pouvoir d'achat}

20 Ainsi, pour justifier la nécessité absolue d'un SMIC, 'surfant' sur l'angoisse qu'ont les Allemands de perdre leur pouvoir d'achat (voir dans ce numéro l'analyse de Stefanie Wahl), les études sur la paupérisation des Allemands fleurissent depuis quelque temps. Le caractère peu fondé des analyses quelque peu fallacieuses avancées pour expliquer des statistiques en soi incontestables commençant à être percé à jour par l'opinion (voir REA 87/08), le mouvement syndical s'empare aujourd'hui des comparaisons internationales pour contrer le principal argument économique des adversaires du SMIC légal : l'effet néfaste sur l'emploi.

\section{Le cas français, instrumentalisé dans les débats}

21 Le cas de la Grande-Bretagne, considéré à l'unanimité comme positif ou, à l'inverse, comme peu pertinent du fait des grandes différences structurelles au niveau de la régulation sociale, ayant été amplement étudié, c'est désormais sur celui de la France que se concentre l'intérêt des Allemands. En guise de réplique à l'analyse impitoyable des effets sur l'emploi, notamment des jeunes, du SMIC français établie dès le rapport annuel 2002/03 par le Conseil des Sages (l'idée d'une transposition à l'Allemagne commençait alors à germer) et qui sert de référence aux adversaires du SMIC allemand, l'institut IMK vient de publier une étude pour démontrer que la France est bien au contraire un exemple à suivre. L'étude, intitulée: "La France, un modèle pour l'Allemagne?" a été publiée dans le numéro de septembre de la revue IMK Report de cet institut créé par un transfuge du DIW de Berlin et proche de la Fondation Hans 
Boeckler, le think tank du DGB. La conclusion du résumé allemand de l'étude laisse perplexe : «La meilleure performance [économique; IB] de la France révèle que la création d'emplois n'est pas obligatoirement liée à la modération salariale ». A suivre... (IB)

INDEX

Mots-clés : activité professionnelle, AEntG, amendement, Arbeitnehmer-Entsendegesetz, CDU, CSU, emploi, loi sur les conditions de travail minimales, loi sur les travailleurs détachés, marché du travail, Mia, projet de loi, salaire minimum, SMIC légal, SPD, syndicat 